\title{
One Learning and Teaching Tool for Preliminary Virtual Instrument Course
}

\author{
Yongkai Fan ${ }^{1}$, Tianze Sun ${ }^{1}$, Xiaolong Fu ${ }^{1}$, Jun $\mathrm{Lin}^{2}$, and Yangyi Sui ${ }^{2}$ \\ ${ }^{1}$ Computer and Information Management Center, Tsinghua University, Beijing 100084, \\ Haidian District, China \\ \{fyk, stz, fuxiaolong\} @ic.tsinghua.edu.cn \\ ${ }^{2}$ Instrument Science and Electronic Engineering College, Jilin University, Changchun 130026, \\ Jilin, China \\ \{lin_jun, syy\}@jlu.edu.cn
}

\begin{abstract}
This paper presents one teaching and leaning assistant tool with the purpose of reinforcing both teaching process and learning process for introductory virtual instrument course. This tool has two main ends: from the teacher's viewpoint, the tool gives preliminary introductory information and foundational theory related the course which will help reinforce lecture and laboratory session, therefore, teacher can save time to pay more attention to personalized education for different student; from the students' viewpoint, it providing a leaning environment which equipped with help, collaborative learning group, communication tool, all of those which can facilitate their personal work and make them more motivated to involved in course session.
\end{abstract}

Keywords: Teaching and leaning assistant tool; Preliminary Virtual Instrument Course; Leaning environment.

\section{Introduction}

The importance of virtual instrument in measurement and instrument filed results in it as a compulsory course appeared in engineering students' curriculum. Virtual instrument programming environment, tool, and even graphic languages rush to support the process of learning virtual instrument. However, students still find learning and programming a virtual instrument is a difficult subject, especially for the beginner. It is said that practice is the best "teacher" for beginner. This Paper describes an experimentation practice platform to meet the needs. The platform is such an e-learning platform for providing test and measurement experimentation, which is based on some distributed measurement hardware resources, and for providing experience sharing means. The end of this platform is to providing student one tool to study virtual instrument and teacher one tool to teaching. This platform consists of four parts: the manager server, the web server, experiment resources, and client software for students. The platform is described in terms of its architecture and an example is illustrated. Further work is outlined in corresponding parts and the conclusions are given in the end. 


\section{Platform Architecture}

The architecture of e-learning platform is showed in Fig. 1. The architecture is mainly composed of four parts: the manager server, the web server, experiment resources, client software and other accessories part.

The manager server: special software runs on the manager server act as a connector between client software and experiment resources. It is the vital part of the e-learning platform, which acts as the gap between client software and experiment resources. It hides the technical details from the users and provides users a transparent connection to experiment resources. On the one hand, the manager server software receives experiment resources registration information and stored information into database. On the other hand, the manager server software broad those experiment resources' information when new experiment resource joining in or the manager server send those experiment resources' information when a user connect to it. Also, the manager server software is a central server responsible for responding to events, such as registration, requiring, data receiving, translating, dispatching and etc, and performs the controlling functionalities and manipulating the underlying logic connection. The screen snapshot is shown as fig 2 .

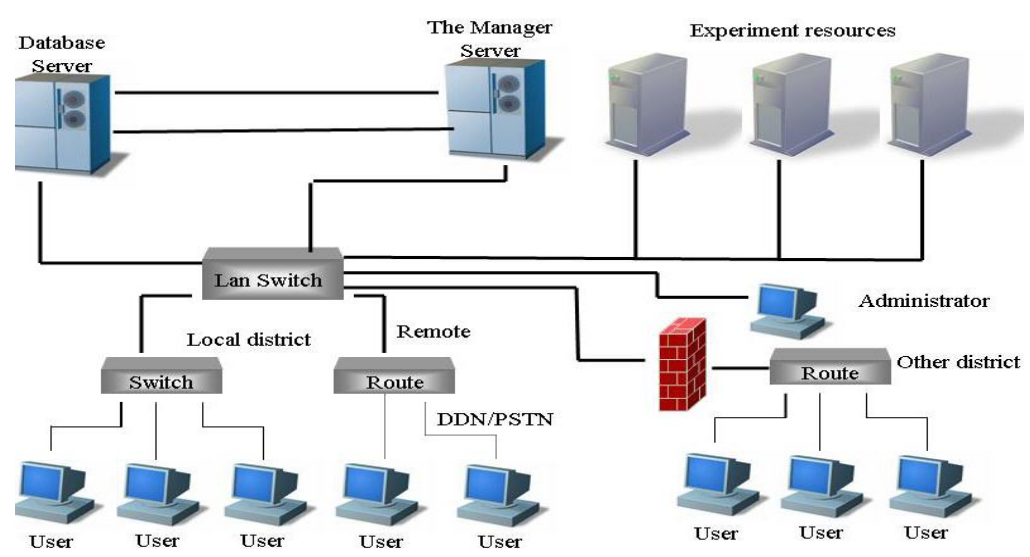

Fig. 1. The architecture of e-learning platform. The web server and the software for manager server share same computer.

Experiment resources: experiment resource is an extensive concept. It refers instrument-equipped computer. The most common used measurement instrument is one data acquisition device. Various sensors connected to the data acquisition device can be treated as one type of experiment resource. The instrument equipped computers are directly connected to instruments in order to measure physical quantities. From the manager server's viewpoint, different experiment resource is the different computer connection. Experiment resources can be located everywhere on the network, but physical connections and access authorizations are given to every legal user.

Client software: with the intention of offering greater convenience to the user, including three different levels user (on-campus user, off-campus users, and distance 
users), client software offers user a friendly and convenient entry to experiment resources. This allows any user to easily install client software in his client computer, connect international network and then can access the instrumentation components (data acquisition devices) from the experiment resources without taking any pains.

The web server: the web server mainly possesses two functions. The first one is electronic forum in which users, whether on campus, off campus or distance user, can login and join to discuss problem they meted met during the process of experiment, the second one, also is the most important one, is sharing experience and normal knowledge. The web server is an assistant for user who wants to get some instruction or documents.

Others: There are special users who are responsible for the maintaining and managing the platform or experiment resources presented as administrator in this architecture, the administrator run corresponding administrator software to monitor and mange the e-learning platform. Firewall and gateway is used to protect server, user operations are controlled through verification of the password or the IP address either of the client or the gateway through which the client connects to the server; Database server is deployed for modeling and storing information needed.

\begin{tabular}{|c|c|c|c|c|}
\hline \multicolumn{2}{|c|}{ 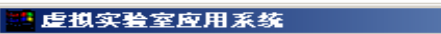 } & & \multicolumn{2}{|c|}{$-|\square| x$} \\
\hline \multicolumn{5}{|l|}{ Eile 브elp } \\
\hline 设备名称 & 设备状棬 & 使用者 & \multicolumn{2}{|l|}{ 备䄇 } \\
\hline gh5105 & 设备可用 & 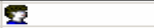 & \multicolumn{2}{|l|}{ 数据采集系统 } \\
\hline phoo2 & 设备可用 & 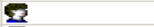 & \multicolumn{2}{|l|}{ 数据采集家统 } \\
\hline LGOO1 & 设备可用 & $\overline{\underline{z}}$ & \multicolumn{2}{|l|}{ 逻轾分析设备 } \\
\hline$\square$ LGOO2 & 设备可用 & $\bar{z}$ & \multicolumn{2}{|l|}{ 逻辑分析设备 } \\
\hline WNOD1 & 设备可用 & $\overline{8}$ & \multicolumn{2}{|l|}{ 存fi者示波哭设备 } \\
\hline W woo2 & 设备可用 & $\overline{2}$ & \multicolumn{2}{|l|}{ 存fi者示波器设备 } \\
\hline \multicolumn{4}{|l|}{4} & 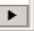 \\
\hline \multicolumn{4}{|c|}{ 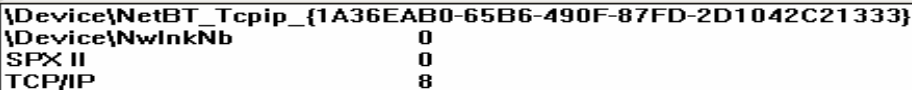 } & - \\
\hline
\end{tabular}

Fig. 2. Screen snapshot of software run on the manager server

\section{Process of Doing Experiment and Experience Sharing}

To explain the practice process, a simple measurement process is provided by a stepby-step explanation.

1. Firstly, the platform's database should start up and prepare for the whole system, the software running on manager server initializes itself and gets ready to receive event from client connection or experiment resources connection.

3. When experiment resources are ready for providing instrument connection, they advertise their IP address and functionalities to the manager server for registration.

4. A user with client software runs client software on his/her own personal computer in which a client workbench is presented on screen, showing in fig. 3 .

5. The manager server will receive a login-request event with a data packet in which user's information and then begin verify process. If authorized, user can select a data acquisition component, for example, on receiving this packet, the manager 
server first assure user's access privilege to the experiment resource to which user want and then decided whether sending user's request to homogenous experiment resource or declining user's request with reasons.

6. When an experiment resource server received a request, it will accept and build a connection with the user. After acquiring data from equipped measurement instrument, experiment resource delivers the sampled data directly to the user who send request. User can do some analysis and process through corresponding software components.

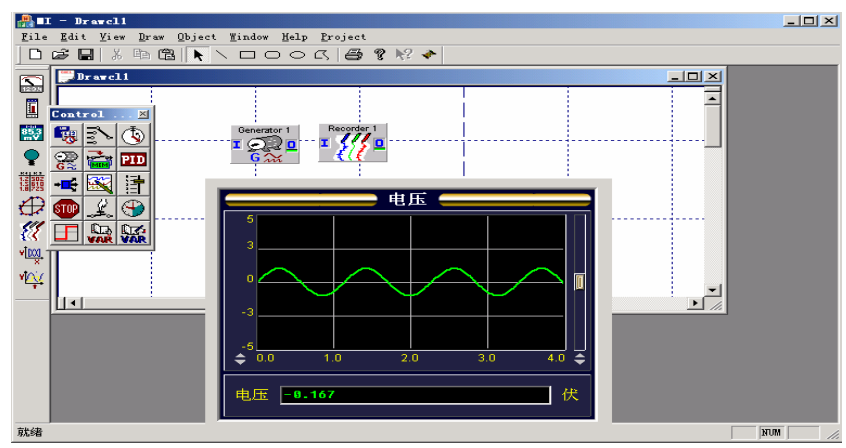

Fig. 3. Screen snapshot of client workbench

\section{Evaluation and Conclusion}

Hands-on experiments have become an important consideration in measurement technologies education. In this paper, one e-learning platform based on distributed measurement hardware resources is presented for education and experience sharing purposes. Though there are many problems, which were found in test phase, should be tackled with, the platform as an accessory experimentation environment provides an effective approach for measurement technologies education according to the test results from individual students. Also, the comparison result of studying effect between students who use this tool before attending virtual instrument class and students who without using this tool indicates that this tool is effective. 